\title{
Fibrations on four-folds with trivial canonical bundles*
}

\author{
Justin Sawon
}

April, 2009

\begin{abstract}
Four-folds with trivial canonical bundles are divided into six classes according to their holonomy group. We consider examples that are fibred by abelian surfaces over the projective plane. We construct such fibrations in five of the six classes, and prove that there is no such fibration in the sixth class. We classify all such fibrations whose generic fibre is the Jacobian of a genus two curve.
\end{abstract}

\section{Introduction}

A holomorphic symplectic four-fold $X$ is a compact Kähler four-fold admitting a holomorphic two-form $\sigma \in \mathrm{H}^{0}\left(X, \Omega^{2}\right)$ which is non-degenerate, i.e., $\sigma^{\wedge 2}$ trivializes the canonical bundle $K_{X}=\Omega^{4}$. The Calabi-Yau metric on $X$ has holonomy group contained in $\mathrm{Sp}(2)$. We say that $X$ is irreducible if it is simply-connected and $\sigma$ is unique up to scale; in this case the holonomy group is equal to $\operatorname{Sp}(2)$. Matsushita [17, 18, 19] showed that if a projective irreducible holomorphic symplectic four-fold $X$ admits a fibration then the generic fibre must be an abelian surface and the base must be isomorphic to $\mathbb{P}^{2}$ (see also Markushevich [15] for the statement about the base).

In the opposite direction, one might ask: "when is a fibration $X \rightarrow \mathbb{P}^{2}$ by abelian surfaces an irreducible holomorphic symplectic four-fold?" (We mean here a fibration whose generic fibre is an abelian surface; we always allow singular fibres.) A necessary condition is that the canonical bundle $K_{X}$ of $X$ must be trivial. Now there is a formula to compute $K_{X}$ due to Fujino and Mori 8$]$ which generalizes Kodaira's formula for the canonical bundle of an elliptic surface; this could be used to check whether $K_{X}$ is trivial. However, this is not enough: as we show in this article, there are numerous examples of four-folds with trivial canonical bundles admitting fibrations by abelian surfaces over $\mathbb{P}^{2}$ which are not irreducible holomorphic symplectic four-folds.

Four-folds with trivial canonical bundles admit Calabi-Yau metrics; they can then be divided into six classes according to the restricted holonomy group of

*2000 Mathematics Subject Classification. 14J35; 14D06, 14J32, 53C26. 
this metric. Irreducible holomorphic symplectic four-folds have holonomy $\operatorname{Sp}(2)$; four of the other classes also contain examples of fibrations by abelian surfaces over $\mathbb{P}^{2}$.

Theorem 1 1. Let $G$ be the trivial group, $\mathrm{SU}(2), \mathrm{SU}(3), \mathrm{SU}(4)$, or $\mathrm{Sp}(2)$. Then there exist four-folds $X$ with $K_{X}$ trivial and holonomy $G$, that are fibred by abelian surfaces over $\mathbb{P}^{2}$.

2. There does not exist a four-fold $X$ with $K_{X}$ trivial and holonomy $\mathrm{SU}(2) \times$ $\mathrm{SU}(2)$, that is fibred by abelian surfaces over $\mathbb{P}^{2}$.

The six different classes are treated in Sections 3 to 8; part two of the theorem is proved in Section 5 (Corollary 16] and Proposition 17) and the examples are constructed in the other sections.

In addition, suppose that $X$ is fibred by Jacobians of genus two curves (roughly this means that the fibres are principally polarized abelian surfaces). Following ideas of Markushevich [14, we classify such fibrations.

Theorem 2 Let $\mathcal{C} \rightarrow \mathbb{P}^{2}$ be a family of genus two curves with 'mild degenerations' (defined in Section 9), and suppose that the compactified relative Jacobian

$$
X:=\overline{\operatorname{Jac}}^{0}\left(X / \mathbb{P}^{2}\right)
$$

has trivial canonical bundle. Then $X$ is either

1. a Calabi-Yau four-fold, with holonomy SU(4), contained in a connected 75-dimensional family,

2. or an irreducible holomorphic symplectic four-fold, with holonomy $\operatorname{Sp}(2)$, contained in a connected 19-dimensional family.

The proof appears in Section 9 (Propositions 21, 22. and 23). Markushevich 14 proved that the holomorphic symplectic four-folds arising in case two are precisely the Beauville-Mukai integrable systems [2], i.e., the family of curves $\mathcal{C} \rightarrow \mathbb{P}^{2}$ must be a complete linear system of genus two curves on a K3 surface. We include a slight simplification of his argument here for completeness. Markushevich's theorem has been generalized to fibrations on higher-dimensional holomorphic symplectic manifolds by the author [26].

In [23, 25] the author described twisted Fourier-Mukai transforms between Lagrangian fibrations $X \rightarrow \mathbb{P}^{n}$ on holomorphic symplectic manifolds and their dual fibrations $\widehat{X} \rightarrow \mathbb{P}^{n}$. The twisting is by a gerbe, which lies in the analytic Brauer group $\mathrm{H}^{2}\left(\widehat{X}, \mathcal{O}^{*}\right)$. For holomorphic symplectic manifolds, this group is connected. Similarly, one could construct twisted Fourier-Mukai transforms between the fibrations constructed in this article and their duals; this may reveal new phenomena, since the Brauer group is discrete in some cases.

The author would like to thank Rick Miranda and Jason Starr for useful conversations, and Markushevich whose papers [14, 15] provided the basis for much of this work (particularly Section 9). The author is grateful for the hospitality of the Korea Institute for Advanced Studies where parts of this article were completed. 


\section{Four-folds with trivial canonical bundles}

\subsection{Holonomy classification}

We will study fibrations over the projective plane whose generic fibres are abelian surfaces and whose total spaces have trivial canonical bundles.

Definition Throughout this article $X$ will denote a compact Kähler manifold of dimension four, with trivial canonical bundle $K_{X} \cong \mathcal{O}_{X}$. We will assume

1. there is a surjective morphism $\pi: X \rightarrow \mathbb{P}^{2}$ with connected fibres,

2. the generic fibre $X_{t}:=\pi^{-1}(t)$ is an abelian surface,

3. $\pi: X \rightarrow \mathbb{P}^{2}$ is a flat fibration, and in particular equidimensional,

4. there exists a smooth section $s: \mathbb{P}^{2} \rightarrow X$ of $\pi$.

Remark It would be desirable to relax the last two assumptions. In principal, given a fibration $X \rightarrow \mathbb{P}^{2}$ that does not admit a section, there should be an associated fibration $X^{0} \rightarrow \mathbb{P}^{2}$ that does admit a section such that $X$ and $X^{0}$ are locally isomorphic as fibrations. Over smooth fibres, $X^{0}$ is the relative Albanese of $X / \mathbb{P}^{2}$, or equivalently the "double-dual", i.e., relative Picard scheme of the relative Picard scheme of $X / \mathbb{P}^{2}$. In practice, the existence of singular fibres makes the construction of $X^{0}$ from $X$ difficult (though not impossible: see $[23,25]$ ).

Lemma 3 If $X$ satisfies the above assumptions then $X$ is projective.

Proof When $X$ is a holomorphic symplectic four-fold, this follows from an argument of Campana and Oguiso (Proposition 3.2 in [21]; see also Lemma 2 in [27]). The same argument works in this more general setting.

Since $X$ is compact and Kähler, with trivial canonical bundle, Yau's Theorem implies that $X$ admits a Ricci-flat metric $g$. The restricted holonomy $\mathrm{Hol}^{0}(g)$ is the group of holonomy maps obtained by parallel transport around nullhomotopic paths. Since $g$ is Ricci-flat, $\operatorname{Hol}^{0}(g)$ must be contained in $\mathrm{SU}(4)$ and Berger's classification of holonomy groups (see Theorem 3.4.1 of Joyce [1]) yields the following six classes.

Proposition 4 The restricted holonomy $\operatorname{Hol}^{0}(g)$ of the Ricci-flat metric on $X$ must be one of the following:

1. the trivial group; there is a finite unramified cover $\tilde{X}$ of $X$ such that $\tilde{X}$ is an abelian four-fold,

2. $\mathrm{SU}(2)$; there is a finite unramified cover $\tilde{X}$ of $X$ such that $\tilde{X}=A \times S$, where $A$ and $S$ are abelian and $K 3$ surfaces respectively,

3. $\mathrm{SU}(2) \times \mathrm{SU}(2)$; either $X$ itself or an unramified double cover $\tilde{X}$ of $X$ is the product $S_{1} \times S_{2}$ of two K3 surfaces, 
4. $\mathrm{SU}(3)$; there is a finite unramified cover $\tilde{X}$ of $X$ such that $\tilde{X}=E \times Y$, where $E$ is an elliptic curve and $Y$ is a Calabi-Yau three-fold,

5. SU(4); $X$ is a Calabi-Yau four-fold,

6. $\mathrm{Sp}(2) ; X$ is an irreducible holomorphic symplectic four-fold.

Proof As mentioned above, the possibilities for the restricted holonomy group follow from Berger's classification. Moreover, the Bogomolov Decomposition Theorem [3] (see also Proposition 6.2.2 of Joyce [11]) states that $X$ admits a finite unramified cover $\tilde{X}$ which is a product manifold

$$
\tilde{X}=T \times Y_{1} \times \ldots \times Y_{p} \times Z_{1} \times \ldots \times Z_{q} .
$$

In this decomposition, $T$ is a flat complex torus (necessarily an abelian variety since $X$ is projective), the $Y_{i}$ s are Calabi-Yau manifolds with holonomy $\operatorname{SU}\left(n_{i}\right)$, and the $Z_{i}$ s are irreducible holomorphic symplectic manifolds with holonomy $\operatorname{Sp}\left(m_{i}\right)$. The small dimensions lead to the six possibilities listed above.

To prove $\tilde{X}=X$ in classes 5 and 6 , and either $\tilde{X}=X$ or $\tilde{X}$ is a double cover of $X$ in class 3 , we compare $\chi\left(\mathcal{O}_{X}\right)$ to $\chi\left(\mathcal{O}_{\tilde{X}}\right)$. If $\tilde{X} \rightarrow X$ is an unramified $d$-to-1 cover then

$$
\chi\left(\mathcal{O}_{\tilde{X}}\right)=d \chi\left(\mathcal{O}_{X}\right)
$$

Moreover, any holomorphic $k$-form on $X$ will lift to a holomorphic $k$-form on $\tilde{X}$, so

$$
h^{k}\left(\mathcal{O}_{\tilde{X}}\right)=h^{0}\left(\Omega_{\tilde{X}}^{k}\right) \geq h^{0}\left(\Omega_{X}^{k}\right)=h^{k}\left(\mathcal{O}_{X}\right)
$$

for all $k$. We also have $h^{4}\left(\mathcal{O}_{X}\right)=h^{0}\left(\Omega_{X}^{4}\right)=1$ since $\Omega_{X}^{4}=K_{X}$ is trivial. One easily concludes that $d$ must equal one in classes 5 and 6 , and must equal one or two in class 3 .

Example We describe a four-fold $X$ with trivial canonical bundle which has $S_{1} \times S_{2}$ as an unramified double cover, as in class 3 . Let $S_{i}$ be a double cover of an Enriques surface $T_{i}$, with covering involution $\gamma_{i}$. Then $\left(\gamma_{1}, \gamma_{2}\right)$ is a fixed-point free involution on $S_{1} \times S_{2}$, and we let

$$
\tilde{X}:=S_{1} \times S_{2} \rightarrow X:=S_{1} \times S_{2} /\left\langle\left(\gamma_{1}, \gamma_{2}\right)\right\rangle .
$$

Denote by $\sigma_{i}$ the holomorphic symplectic form on $S_{i}$, and by $p_{i}^{*} \sigma_{i}$ the pull-back to $\tilde{X}=S_{1} \times S_{2}$, where $p_{i}$ is projection to the $i$ th factor. Then $\gamma_{i}$ acts on $\sigma_{i}$ by -1 , so $\left(\gamma_{1}, \gamma_{2}\right)$ acts on $p_{i}^{*} \sigma_{i}$ by -1 . Neither two-form $p_{i}^{*} \sigma_{i}$ descends to $X$, but their product $p_{1}^{*} \sigma_{1} \wedge p_{2}^{*} \sigma_{2}$ does descend to give a trivialization of $K_{X}$.

We conclude that

$$
h^{k}\left(\mathcal{O}_{X}\right)= \begin{cases}1 & \text { if } k=0 \text { or } 4, \\ 0 & \text { otherwise. }\end{cases}
$$

If the holonomy group $\operatorname{Hol}(g)$ of $X$ were the same as the restricted holonomy group $\mathrm{Hol}^{0}(g) \cong \mathrm{SU}(2) \times \mathrm{SU}(2)$ then there would be parallel (and hence holomorphic) two-forms on $X$; but $h^{0}\left(\Omega_{X}^{2}\right)=h^{2}\left(\mathcal{O}_{X}\right)=0$. Therefore $\operatorname{Hol}(g)$ is 
strictly bigger than $\operatorname{Hol}^{0}(g)$. On the other hand, there is a surjective group homomorphism

$$
\pi_{1}(X) \cong \mathbb{Z} / 2 \mathbb{Z} \rightarrow \operatorname{Hol}(g) / \operatorname{Hol}^{0}(g)
$$

(see Proposition 2.2.6 of Joyce [1]). An example of such a group is

$$
\operatorname{Hol}(g)=\left\{\left(\begin{array}{cc}
A & 0 \\
0 & B
\end{array}\right) \mid A, B \in \mathrm{U}(2), \operatorname{det} A=\operatorname{det} B= \pm 1\right\} \subset \mathrm{SU}(4) .
$$

Remark It is enough that just one of the involutions $\gamma_{i}$ be fixed-point free, for then $\left(\gamma_{1}, \gamma_{2}\right)$ will be fixed-point free. For example, $S_{1}$ could be the unramified double cover of an Enriques surface $T_{1}$ and $S_{2}$ could be the double cover of $\mathbb{P}^{2}$ ramified over a sextic.

This construction exhibits all the possibilities in class 3 , as we now show.

Proposition 5 Let $X$ be a four-fold with trivial canonical bundle which has the product $S_{1} \times S_{2}$ of two K3 surfaces as an unramified double cover. Then $X \cong S_{1} \times S_{2} /\left\langle\left(\gamma_{1}, \gamma_{2}\right)\right\rangle$, with $\gamma_{1}$ the covering involution of the unramified double cover $S_{1} \rightarrow T_{1}$ of an Enriques surface and $\gamma_{2}$ the covering involution of some other (possibly ramified) double cover $S_{2} \rightarrow T_{2}$.

Proof Let $\gamma$ be the covering involution of $\tilde{X}=S_{1} \times S_{2} \rightarrow X$. Since $\chi\left(\mathcal{O}_{X}\right)=$ $\frac{1}{2} \chi\left(\mathcal{O}_{\tilde{X}}\right)=2$ and $h^{k}\left(\mathcal{O}_{X}\right) \leq h^{k}\left(\mathcal{O}_{\tilde{X}}\right)$ we must have

$$
h^{k}\left(\mathcal{O}_{X}\right)= \begin{cases}1 & \text { if } k=0 \text { or } 4 \\ 0 & \text { otherwise }\end{cases}
$$

In particular, the involution $\gamma$ does not fix either two-form $p_{i}^{*} \sigma_{i}$, so it must act as -1 .

Let $x_{2} \in S_{2}$. By integrating $p_{1}^{*} \sigma_{1} \bar{\sigma}_{1}$ over $S_{1} \times x_{2}$ and $\gamma\left(S_{1} \times x_{2}\right)$ we see that $\gamma$ takes $S_{1} \times x_{2}$ to $S_{1} \times y_{2}$ for some $y_{2} \in S_{2}$ (these are calibrated submanifolds for $\left.p_{1}^{*} \sigma_{1} \bar{\sigma}_{1}\right)$. Similarly $x_{1} \times S_{2}$ is sent to $y_{1} \times S_{2}$, and we conclude that $\gamma=\left(\gamma_{1}, \gamma_{2}\right)$ acts independently on each factor. If $\gamma_{i}$ both have fixed-points $p_{i}$, then $\left(\gamma_{1}, \gamma_{2}\right)$ would have a fixed-point $\left(p_{1}, p_{2}\right)$, which is impossible. Therefore $\gamma_{1}$, say, is fixed-point free and the quotient $S_{1} /\left\langle\gamma_{1}\right\rangle$ is an Enriques surface.

\subsection{An abelian fibration on $\tilde{X}$}

Although we want to understand fibrations $X \rightarrow \mathbb{P}^{2}$, it is sometimes easier to work with the space $\tilde{X}$ because of the product decompositions it admits in the different classes. Of course $\tilde{X}$ is also a fibration over $\mathbb{P}^{2}$ by virtue of the composition of maps $\tilde{X} \rightarrow X \rightarrow \mathbb{P}^{2}$. Let $\Gamma$ be the order $d$ covering group of $\tilde{X} \rightarrow X$; then $\Gamma$ is isomorphic to the quotient group $\pi_{1}(X) / \pi_{1}(\tilde{X})$. Let $t$ be a generic point of $\mathbb{P}^{2}$. The generic fibre $A:=\pi^{-1}(t)$ of $\pi: X \rightarrow \mathbb{P}^{2}$ is an abelian surface, and hence the generic fibre $\tilde{A}:=\tilde{\pi}^{-1}(t)$ of $\tilde{\pi}: \tilde{X} \rightarrow \mathbb{P}^{2}$ will be an unramified cover of $A$ with covering group $\Gamma$. It follows that $\tilde{A}$ must be a disjoint union of abelian surfaces. Let $\Gamma_{0} \subset \Gamma$ be the subgroup of elements 
which do not permute the connected components of $\tilde{A}$. A connected component $\tilde{A}_{0}$ of $\tilde{A}$ will be a cover of $A$ with covering group $\Gamma_{0}$; thus $\Gamma_{0}$ is isomorphic to the quotient group $\pi_{1}(A) / \pi_{1}\left(\tilde{A}_{0}\right)$, and in particular it is abelian.

We now take the Stein factorization of $\tilde{\pi}: \tilde{X} \rightarrow \mathbb{P}^{2}$, which gives

$$
\begin{array}{ccc}
\tilde{X} & \stackrel{\phi}{\rightarrow} & Z \\
\downarrow & & \downarrow \tau \\
X & \rightarrow & \mathbb{P}^{2}
\end{array}
$$

where the generic fibre of $\phi$ is an abelian surface, such as $\tilde{A}_{0}$. Moreover, $\tau: Z \rightarrow$ $\mathbb{P}^{2}$ is a Galois cover with Galois group $\Gamma / \Gamma_{0}$; the points of $\tau^{-1}(t)$ correspond to the different connected components of $\tilde{A}$, and $\Gamma / \Gamma_{0}$ acts freely and transitively on these components. In particular, $Z \rightarrow \mathbb{P}^{2}$ is a $d^{\prime}$-to- 1 cover, where $d^{\prime}$ divides $d$. (This illustrates the utility of the restrictions that $d$ is one in classes 5 and 6 , and one or two in class 3.)

The problem of constructing examples can be formulated as follows. Given $\tilde{X}$ from one of the six classes, we look for a fibration of $\tilde{X}$ by abelian surfaces over a surface $Z$. We try to express $Z$ as a Galois cover $\tau: Z \rightarrow \mathbb{P}^{2}$, with Galois group $\Gamma / \Gamma_{0}$. Of course $\tau$ must be ramified since $\mathbb{P}^{2}$ is simply connected. There will be a quotient $\tilde{X} / \Gamma_{0}$ which is also fibred by abelian surfaces over $Z$. The problem is to extend the action of $\Gamma / \Gamma_{0}$ on $Z$, which has fixed-points, to a fixed-point free action on $\tilde{X} / \Gamma_{0}$; for then the quotient

$$
\left(\tilde{X} / \Gamma_{0}\right) /\left(\Gamma / \Gamma_{0}\right) \cong \tilde{X} / \Gamma
$$

is the required four-fold $X$, fibred by abelian surfaces over $\mathbb{P}^{2}$. The following diagram summarizes this construction.

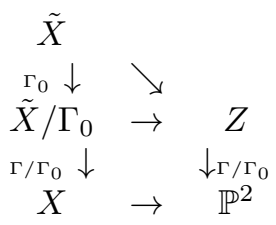

If some element of $\Gamma / \Gamma_{0}$ fixes $s \in Z$, then that element must act in a fixedpoint free manner on the abelian surface fibre above $s$. Usually the quotient of this action will again be an abelian surface, and thus the element should act by a translation. This suggests that $\Gamma / \Gamma_{0}$ should be abelian, and we will keep this as a guiding principle when constructing examples.

However, there may not be a point $s \in Z$ fixed by the entire group $\Gamma / \Gamma_{0}$, so we won't find necessarily find that the entire group acts as translations on a single abelian surface. Moreover, $s$ could lie in the discriminant locus of the abelian surface fibration, implying that the fibre above $s$ is a degeneration rather than a smooth abelian surface.

\subsection{Direct image sheaves}

Returning to $\pi: X \rightarrow \mathbb{P}^{2}$, we have the following results concerning direct images of the structure sheaf. 
Lemma 6 The sheaves $R^{0} \pi_{*} \mathcal{O}_{X}, R^{1} \pi_{*} \mathcal{O}_{X}$, and $R^{2} \pi_{*} \mathcal{O}_{X}$ on $\mathbb{P}^{2}$ are locally free.

Proof Theorem 2.1 of Kollár [12] says that $R^{i} \pi_{*} K_{X}$ is torsion-free for $i \geq 0$ (and vanishes for $i>2$ ), but $K_{X} \cong \mathcal{O}_{X}$ since $X$ has trivial canonical bundle. Corollary 3.9 of Kollár [13] then says that in our case $R^{i} \pi_{*} \mathcal{O}_{X}$ is reflexive, but reflexive sheaves on surfaces are locally free. Note that $R^{1} \pi_{*} \mathcal{O}_{X}$ has rank two, while $R^{0} \pi_{*} \mathcal{O}_{X}$ and $R^{2} \pi_{*} \mathcal{O}_{X}$ are line bundles.

Lemma 7 We have $R^{0} \pi_{*} \mathcal{O}_{X} \cong \mathcal{O}_{\mathbb{P}^{2}}$ and $R^{2} \pi_{*} \mathcal{O}_{X} \cong \mathcal{O}_{\mathbb{P}^{2}}(-3)$.

Proof Since $\pi: X \rightarrow \mathbb{P}^{2}$ has connected fibres, $R^{0} \pi_{*} \mathcal{O}_{X} \cong \mathcal{O}_{\mathbb{P}^{2}}$ is automatic. Since $X$ has trivial canonical bundle, we have $h^{4}\left(X, \mathcal{O}_{X}\right)=1$. This number can also be computed using the Leray spectral sequence for $X \rightarrow \mathbb{P}^{2}$

$$
E_{2}^{p, q}:=\mathrm{H}^{q}\left(\mathbb{P}^{2}, R^{p} \pi_{*} \mathcal{O}_{X}\right) \Rightarrow \mathrm{H}^{p+q}\left(X, \mathcal{O}_{X}\right) .
$$

Thus we have

$$
\mathrm{H}^{4}\left(X, \mathcal{O}_{X}\right) \cong \mathrm{H}^{2}\left(\mathbb{P}^{2}, R^{2} \pi_{*} \mathcal{O}_{X}\right) \cong \mathrm{H}^{0}\left(\mathbb{P}^{2},\left(R^{2} \pi_{*} \mathcal{O}_{X}\right)^{*} \otimes K_{\mathbb{P}^{2}}\right)^{*}
$$

implying that $R^{2} \pi_{*} \mathcal{O}_{X} \cong \mathcal{O}_{\mathbb{P}^{2}}(-3)$.

Lemma 8 Let $V$ denote the rank two bundle $R^{1} \pi_{*} \mathcal{O}_{X}$. Then

$$
\mathrm{H}^{q}\left(\mathbb{P}^{2}, V\right) \cong \mathrm{H}^{q+1}\left(X, \mathcal{O}_{X}\right)
$$

for all $q$.

Proof The previous lemma implies that the Leray spectral sequence degenerates at $E_{2}^{p, q}$, and hence

$$
\mathrm{H}^{q}\left(\mathbb{P}^{2}, R^{p} \pi_{*} \mathcal{O}_{X}\right) \cong \mathrm{H}^{p+q}\left(X, \mathcal{O}_{X}\right) .
$$

Remark Recall that

$$
h^{i}\left(X, \mathcal{O}_{X}\right)=h^{0}\left(X, \Omega_{X}^{i}\right) \leq h^{0}\left(\tilde{X}, \Omega_{\tilde{X}}^{i}\right)
$$

because any holomorphic form on $X$ must lift to $\tilde{X}$. The right hand side can easily be calculated in each of the six classes. We also have

$$
\chi\left(\mathcal{O}_{\tilde{X}}\right)=d \chi\left(\mathcal{O}_{X}\right)
$$

and $h^{3}\left(X, \mathcal{O}_{X}\right)=h^{1}\left(X, \mathcal{O}_{X}\right)$ because of Serre duality and the triviality of the canonical bundle.

There are therefore a limited number of possibilities for $h^{i}\left(X, \mathcal{O}_{X}\right)$ in each of the six classes, and these lead to a limited number of possibilities for the rank two bundle $V$, which we will explore in the following sections. 


\section{$3 \quad \tilde{X}$ an abelian four-fold}

There are four possibilities for $\left(h^{q}\left(\mathbb{P}^{2}, V\right)\right)_{q=0}^{2}=\left(h^{i}\left(X, \mathcal{O}_{X}\right)\right)_{i=1}^{3}$, namely

$$
(4,6,4), \quad(3,4,3), \quad(2,2,2), \quad \text { or } \quad(1,0,1) .
$$

Lemma 9 In the first subcase $X$ is an abelian four-fold. However, an abelian four-fold $X$ cannot admit a fibration by abelian surfaces over $\mathbb{P}^{2}$.

Proof If $h^{0}\left(X, \Omega_{X}^{1}\right)=h^{1}\left(X, \mathcal{O}_{X}\right)=4$, then all holomorphic one-forms on $\tilde{X}$ must be invariant under the action of the covering group of $\tilde{X} \rightarrow X$, so that they descend to $X$. Moreover, the Albanese variety $A$ of $X$ will be an abelian four-fold and the composition

$$
\tilde{X} \rightarrow X \rightarrow A
$$

of the covering and Albanese maps will be an isogeny. This forces $\tilde{X} \rightarrow X$ to also be an isogeny, and hence $X$ is an abelian four-fold.

Suppose there is a fibration on $X$ whose generic fibre is an abelian surface $B \subset X$. Then $B$ must be the translation of an abelian subgroup $B_{0} \subset X$, and the deformations of $B$ in $X$ will be parametrized by the quotient group $X / B_{0}$. In other words, the base of the fibration on $X$ must be an abelian surface $X / B_{0}$, not the projective plane $\mathbb{P}^{2}$.

Lemma 10 The second subcase is impossible: there does not exist a rank two vector bundle $V$ on $\mathbb{P}^{2}$ with

$$
\left(h^{q}\left(\mathbb{P}^{2}, V\right)\right)_{q=0}^{2}=(3,4,3) .
$$

Proof By assumption $V$ admits three independent sections. If these sections don't generate the fibre of $V$ at a generic point, then they define a subbundle; but then $V$ will be a direct sum of line bundles, contradicting $h^{1}\left(\mathbb{P}^{2}, V\right)=4$. Therefore the evaluation map

$$
\mathrm{H}^{0}\left(\mathbb{P}^{2}, V\right) \otimes \mathcal{O}_{\mathbb{P}^{2}} \rightarrow V
$$

is surjective at a generic point; let the kernel be $\mathcal{O}_{\mathbb{P}^{2}}(k)$ with $k \leq 0$ and let the cokernel be the torsion sheaf $\mathcal{F}$. We have a resolution of $\mathcal{F}$

$$
0 \rightarrow \mathcal{O}_{\mathbb{P}^{2}}(k) \rightarrow \mathcal{O}_{\mathbb{P}^{2}}^{\oplus 3} \rightarrow V \rightarrow \mathcal{F} \rightarrow 0
$$

which can be used to compute the cohomology of $\mathcal{F}$. Many of the terms will vanish; for instance

$$
h^{1}\left(\mathcal{O}_{\mathbb{P}^{2}}(k)\right)=h^{2}\left(\mathcal{O}_{\mathbb{P}^{2}}(k)\right)=h^{1}\left(\mathcal{O}_{\mathbb{P}^{2}}^{\oplus 3}\right)=h^{2}\left(\mathcal{O}_{\mathbb{P}^{2}}^{\oplus 3}\right)=0,
$$

whereas $h^{2}(V)=3$ by hypothesis. This will imply that $h^{2}(\mathcal{F})=3$, a contradiction since $\mathcal{F}$ is a torsion sheaf supported on a subscheme of $\mathbb{P}^{2}$ of dimension at most one. 
Lemma 11 The third subcase is impossible: there does not exist a rank two vector bundle $V$ on $\mathbb{P}^{2}$ with

$$
\left(h^{q}\left(\mathbb{P}^{2}, V\right)\right)_{q=0}^{2}=(2,2,2) .
$$

Proof By assumption $V$ admits two independent sections. As in the proof of Lemma 10, the evaluation map

$$
\mathrm{H}^{0}\left(\mathbb{P}^{2}, V\right) \otimes \mathcal{O}_{\mathbb{P}^{2}} \rightarrow V
$$

is surjective at a generic point; let the cokernel be the torsion sheaf $\mathcal{F}$. Then we have a short exact sequence

$$
0 \rightarrow \mathcal{O}_{\mathbb{P}^{2}}^{\oplus 2} \rightarrow V \rightarrow \mathcal{F} \rightarrow 0
$$

whose corresponding long exact sequence yields

$$
\mathrm{H}^{2}\left(\mathcal{O}_{\mathbb{P}^{2}}^{\oplus 2}\right) \rightarrow \mathrm{H}^{2}(V) \rightarrow \mathrm{H}^{2}(\mathcal{F}) \rightarrow 0 .
$$

The left-most term vanishes, implying that

$$
h^{2}(\mathcal{F})=h^{2}(V)=2 .
$$

This is a contradiction since $\mathcal{F}$ has support of dimension at most one.

Lemma 12 In the fourth subcase $V \cong \mathcal{O}_{\mathbb{P}^{2}} \oplus \mathcal{O}_{\mathbb{P}^{2}}(-3)$.

Proof By assumption $V$ admits a section. Let the cokernel of the evaluation map

$$
\mathrm{H}^{0}\left(\mathbb{P}^{2}, V\right) \otimes \mathcal{O}_{\mathbb{P}^{2}} \rightarrow V
$$

be $\mathcal{O}_{\mathbb{P}^{2}}(k) \otimes \mathcal{I}_{Z}$, where $k=c_{1}(V)$ and $\mathcal{I}_{Z}$ is the ideal sheaf of a zero-dimensional subscheme $Z \subset \mathbb{P}^{2}$. Then we have a short exact sequence

$$
0 \rightarrow \mathcal{O}_{\mathbb{P}^{2}} \rightarrow V \rightarrow \mathcal{O}_{\mathbb{P}^{2}}(k) \otimes \mathcal{I}_{Z} \rightarrow 0
$$

whose corresponding long exact sequence yields

$$
\mathrm{H}^{2}\left(\mathcal{O}_{\mathbb{P}^{2}}\right) \rightarrow \mathrm{H}^{2}(V) \rightarrow \mathrm{H}^{2}\left(\mathcal{O}_{\mathbb{P}^{2}}(k) \otimes \mathcal{I}_{Z}\right) \rightarrow 0 .
$$

The left-most term vanishes, implying that

$$
h^{2}\left(\mathcal{O}_{\mathbb{P}^{2}}(k)\right)=h^{2}\left(\mathcal{O}_{\mathbb{P}^{2}}(k) \otimes \mathcal{I}_{Z}\right)=h^{2}(V)=1 .
$$

Therefore $c_{1}(V)=k=-3$.

Next the Riemann-Roch formula gives

$$
\chi(V)=2 \chi\left(\mathcal{O}_{\mathbb{P}^{2}}\right)+\frac{c_{1}(V)\left(c_{1}(V)+3\right)}{2}-c_{2}(V)
$$

and substituting $\chi(V)=2$ and $c_{1}(V)=-3$ yields $c_{2}(V)=0$. It follows that the zero-dimensional subscheme $Z$ is empty. Moreover, the short exact sequence

$$
0 \rightarrow \mathcal{O}_{\mathbb{P}^{2}} \rightarrow V \rightarrow \mathcal{O}_{\mathbb{P}^{2}}(-3) \rightarrow 0
$$

must split, completing the proof. 
We can construct an example in this fourth subcase.

Example Recall the discussion of Subsection 2.2. The abelian surface fibration on $X$ induces an abelian surface fibration on $\tilde{X}$, with base $Z$. The argument in the proof of Lemma 9 shows that $Z$ must be an abelian surface. First we need to find a Galois cover $Z \rightarrow \mathbb{P}^{2}$ with $Z$ an abelian surface. Then we need to lift the group action to a fixed-point free action on $\tilde{X}$.

Let $E$ be an elliptic curve and let $Z$ be $E \times E$. Since $E$ is a double cover of $\mathbb{P}^{1}$, we get the composition of maps

$$
Z=E \times E \rightarrow \mathbb{P}^{1} \times \mathbb{P}^{1} \rightarrow \operatorname{Sym}^{2} \mathbb{P}^{1} \cong \mathbb{P}^{2} .
$$

This expresses $Z$ as a Galois cover of $\mathbb{P}^{2}$. Let $z_{1}$ and $z_{2}$ be coordinates on the first and second copies of $E$ respectively. Then the Galois group $\Gamma$ is generated by elements $\gamma_{1}, \gamma_{2}$, and $\gamma_{3}$ which act by

$$
\begin{aligned}
& \gamma_{1} \cdot\left(z_{1}, z_{2}\right)=\left(-z_{1}, z_{2}\right), \\
& \gamma_{2} \cdot\left(z_{1}, z_{2}\right)=\left(z_{1},-z_{2}\right), \\
\text { and } \quad & \gamma_{3} \cdot\left(z_{1}, z_{2}\right)=\left(z_{2}, z_{1}\right) .
\end{aligned}
$$

Note that $\gamma_{3} \gamma_{1} \gamma_{3}=\gamma_{2}$ and $\Gamma$ is isomorphic to the dihedral group $D_{8}$ : for instance, the element $\gamma_{1} \gamma_{3}$ corresponds to a rotation of order four.

Next we lift the $\Gamma$-action to the abelian four-fold $\tilde{X}$. For simplicity, we assume that $\tilde{X}$ is $E \times E \times E_{3} \times E_{4}$ where $E_{3}$ and $E_{4}$ are arbitrary elliptic curves. Let $z_{3}$ and $z_{4}$ be coordinates on $E_{3}$ and $E_{4}$ respectively, and write $E_{3} \cong \mathbb{C} /\left\langle 1, \tau_{3}\right\rangle$ and $E_{4} \cong \mathbb{C} /\left\langle 1, \tau_{4}\right\rangle$. We define a $\Gamma$-action on $E_{3} \times E_{4}$ by

$$
\begin{aligned}
\gamma_{1} \cdot\left(z_{3}, z_{4}\right) & =\left(z_{3}+\frac{1}{2},-z_{4}+\frac{1}{4}\right), \\
\gamma_{2} \cdot\left(z_{3}, z_{4}\right) & =\left(z_{3}+\frac{1}{2},-z_{4}+\frac{3}{4}\right), \\
\text { and } \quad \gamma_{3} \cdot\left(z_{3}, z_{4}\right) & =\left(z_{3}+\frac{1}{2} \tau_{3},-z_{4}\right) .
\end{aligned}
$$

The 'rotation' $\gamma_{1} \gamma_{3}$ acts by a translation

$$
\gamma_{1} \gamma_{3} \cdot\left(z_{3}, z_{4}\right)=\left(z_{3}+\frac{1}{2}+\frac{1}{2} \tau_{3}, z_{4}+\frac{1}{4}\right)
$$

of order four. Thus the quotient by $\left\langle\gamma_{1} \gamma_{3}\right\rangle$ is an abelian surface, and further quotienting by $\mathbb{Z}_{2} \cong \Gamma /\left\langle\gamma_{1} \gamma_{3}\right\rangle$ produces a bielliptic surface. Note that every non-trivial element of $\Gamma$ acts in a fixed-point free manner on $E_{3} \times E_{4}$, and hence the product $\Gamma$-action on $\tilde{X}=E \times E \times E_{3} \times E_{4}$ is also fixed-point free. This action preserves the four-form $d z_{1} \wedge d z_{2} \wedge d z_{3} \wedge d z_{4}$; the only other forms it preserves are the one-form $d z_{3}$ and the three-form $d z_{1} \wedge d z_{2} \wedge d z_{4}$. Thus the quotient $X:=\tilde{X} / \Gamma$ has trivial canonical bundle and

$$
\left(h^{i}\left(X, \mathcal{O}_{X}\right)\right)_{i=1}^{3}=(1,0,1) .
$$


If $t$ is a generic point of $\mathbb{P}^{2}$, then the fibre of $Z \rightarrow \mathbb{P}^{2}$ over $t$ consists of eight points, and hence the fibre of $\tilde{X} \rightarrow \mathbb{P}^{2}$ over $t$ consists of eight copies of $E_{3} \times E_{4}$. Since $\Gamma$ acts freely and transitively on these copies of $E_{3} \times E_{4}$, the fibre of $X \rightarrow \mathbb{P}^{2}$ over $t$ will consist of a single copy of $E_{3} \times E_{4}$. Thus $X$ is a generically isotrivial abelian surface fibration over $\mathbb{P}^{2}$ : generic fibres are all isomorphic to the fixed abelian surface $E_{3} \times E_{4}$.

\section{$4 \tilde{X}=A \times S, A$ and $S$ abelian and K3 surfaces}

There are two possibilities for $\left(h^{q}\left(\mathbb{P}^{2}, V\right)\right)_{q=0}^{2}=\left(h^{i}\left(X, \mathcal{O}_{X}\right)\right)_{i=1}^{3}$, namely

$$
(2,2,2) \quad \text { or } \quad(1,0,1) \text {. }
$$

By Lemma 11 there is no rank two vector bundle $V$ on $\mathbb{P}^{2}$ in the first subcase. In particular, if $X$ is $A \times S$ itself, then $X$ cannot be fibred by abelian surfaces over $\mathbb{P}^{2}$. In the second subcase $V \cong \mathcal{O}_{\mathbb{P}^{2}} \oplus \mathcal{O}_{\mathbb{P}^{2}}(-3)$ by Lemma 12 and $X$ must be a non-trivial quotient of $\tilde{X}=A \times S$.

We can construct an example in this second subcase.

Example Recall again the discussion in Subsection 2.2. There should be an induced abelian surface fibration on $\tilde{X}=A \times S$. An obvious choice would be the trivial fibration $\tilde{X} \rightarrow S$. Thus we need to express $S$ as a Galois cover of $\mathbb{P}^{2}$, so we let $S$ be a hyperelliptic K3 surface, i.e., a double cover of $\mathbb{P}^{2}$ branched over a sextic curve. If $\sigma$ is the holomorphic symplectic two-form on $S$, then $\gamma^{*} \sigma=-\sigma$ where $\gamma$ is the covering involution of $S \rightarrow \mathbb{P}^{2}$.

Next we extend the action of $\gamma$ to an involution on $\tilde{X}=A \times S$. The action on $A$ must take the two-form $d z_{1} \wedge d z_{2}$ to $-d z_{1} \wedge d z_{2}$, so that the involution preserves the triviality of the canonical bundle on $\tilde{X}$. Thus we let $A$ be the product of two elliptic curves $E_{1} \cong \mathbb{C} /\left\langle 1, \tau_{1}\right\rangle$ and $E_{2} \cong \mathbb{C} /\left\langle 1, \tau_{2}\right\rangle$ with coordinates $z_{1}$ and $z_{2}$ respectively, and we let $\gamma$ act by

$$
\gamma \cdot\left(z_{1}, z_{2}\right)=\left(z_{1}+\frac{1}{2},-z_{2}\right) .
$$

The quotient $E_{1} \times E_{2} /\langle\gamma\rangle$ is a bielliptic surface. This involution on $E_{1} \times E_{2}$ is fixed-point free, and therefore the product action of $\gamma$ on $\tilde{X}=E_{1} \times E_{2} \times S$ is also fixed-point free. The action preserves the four-form $d z_{1} \wedge d z_{2} \wedge \sigma$, the one-form $d z_{1}$ and the three-form $d z_{2} \wedge \sigma$. Thus the quotient $X:=\tilde{X} /\langle\gamma\rangle$ has trivial canonical bundle and

$$
\left(h^{i}\left(X, \mathcal{O}_{X}\right)\right)_{i=1}^{3}=(1,0,1) .
$$

Over a generic point $t \in \mathbb{P}^{2}$, the fibre of $S \rightarrow \mathbb{P}^{2}$ consists of two points, the fibre of $\tilde{X} \rightarrow \mathbb{P}^{2}$ consists of two copies of $E_{1} \times E_{2}$, and hence the fibre of $X \rightarrow \mathbb{P}^{2}$ will consist of a single copy of $E_{1} \times E_{2}$. This expresses $X$ as a generically isotrivial abelian surface fibration over $\mathbb{P}^{2}$. 


\section{$5 \quad \tilde{X}=S_{1} \times S_{2}, S_{1}$ and $S_{2}$ K3 surfaces}

There are two possibilities for $\left(h^{q}\left(\mathbb{P}^{2}, V\right)\right)_{q=0}^{2}=\left(h^{i}\left(X, \mathcal{O}_{X}\right)\right)_{i=1}^{3}$, namely

$$
(0,2,0) \quad \text { or } \quad(0,0,0) \text {. }
$$

In the first subcase $d$ must equal one, so that $X=\tilde{X}=S_{1} \times S_{2}$. In the second subcase $d$ must equal two, so that $\tilde{X}$ is a double cover of $X$.

The next two lemmas could have been proved earlier, but we did not need them until now; first some notation. Let $\Delta \subset \mathbb{P}^{2}$ be the degeneracy locus which parametrizes singular fibres of $\pi: X \rightarrow \mathbb{P}^{2}$, and let $U=\mathbb{P}^{2} \backslash \Delta$ be its complement. For $t \in U$ we have a canonical isomorphism

$$
\Lambda^{2}\left(R^{1} \pi_{*} \mathcal{O}_{X}\right)_{t}=\Lambda^{2} \mathrm{H}^{1}\left(X_{t}, \mathcal{O}_{X_{t}}\right) \cong \mathrm{H}^{2}\left(X_{t}, \mathcal{O}_{X_{t}}\right)=\left(R^{2} \pi_{*} \mathcal{O}_{X}\right)_{t}
$$

and thus

$$
\left.\left.\Lambda^{2}\left(R^{1} \pi_{*} \mathcal{O}_{X}\right)\right|_{U} \cong R^{2} \pi_{*} \mathcal{O}_{X}\right|_{U}
$$

Lemma 13 There is an inclusion

$$
\Lambda^{2}\left(R^{1} \pi_{*} \mathcal{O}_{X}\right) \hookrightarrow R^{2} \pi_{*} \mathcal{O}_{X}
$$

which extends the above isomorphism. In particular, we have

$$
c_{1}(V)=c_{1}\left(R^{1} \pi_{*} \mathcal{O}_{X}\right) \leq c_{1}\left(R^{2} \pi_{*} \mathcal{O}_{X}\right)=-3 .
$$

Moreover, if either

- $\mathrm{H}^{1}\left(X_{t}, \mathbb{C}\right) \geq 2$ where $X_{t}$ is a generic singular fibre of $X \rightarrow \mathbb{P}^{2}$

- or the monodromy of $R^{1} \pi_{*} \mathcal{O}_{X}$ around $\Delta$ is unipotent

then the inclusion above is actually an isomorphism and hence $c_{1}(V)=-3$.

Proof The proofs of these statements can be found in Matsushita 20, particularly Proposition 2.13 and Section 3. For instance, the inclusion

$$
\Lambda^{2}\left(R^{1} \pi_{*} \mathcal{O}_{X}\right) \hookrightarrow R^{2} \pi_{*} \mathcal{O}_{X}
$$

is a consequence of functoriality of the variation of Hodge structure.

Lemma 14 The higher cohomology of $V(k):=V \otimes \mathcal{O}_{\mathbb{P}^{2}}(k)$ vanishes for $k$ positive, i.e.,

$$
h^{q}\left(\mathbb{P}^{2}, V(k)\right)=0
$$

for $k \geq 1$ and $q \geq 1$.

Proof This is the third part of Theorem 2.1 in Kollár [12.

We now return to the two subcases of this section. 
Lemma 15 The first subcase is impossible: there does not exist a rank two vector bundle $V$ on $\mathbb{P}^{2}$ with

$$
\left(h^{q}\left(\mathbb{P}^{2}, V\right)\right)_{q=0}^{2}=(0,2,0),
$$

$c_{1}(V) \leq-3$, and $h^{q}\left(\mathbb{P}^{2}, V(1)\right)=0$ for $q \geq 1$.

Proof The Riemann-Roch formula gives

$$
\chi(V)=2 \chi\left(\mathcal{O}_{\mathbb{P}^{2}}\right)+\frac{c_{1}(V)\left(c_{1}(V)+3\right)}{2}-c_{2}(V)
$$

whereas we know that $\chi(V)=-2$. Therefore

$$
\begin{aligned}
\chi(V(1)) & =2 \chi\left(\mathcal{O}_{\mathbb{P}^{2}}\right)+\frac{\left(c_{1}(V)+2\right)\left(c_{1}(V)+2+3\right)}{2}-\left(c_{2}(V)+c_{1}(V)+1\right) \\
& =\left(2 \chi\left(\mathcal{O}_{\mathbb{P}^{2}}\right)+\frac{c_{1}(V)\left(c_{1}(V)+3\right)}{2}-c_{2}(V)\right)+c_{1}(V)+4 \\
& =\chi(V)+c_{1}(V)+4 \\
& =c_{1}(V)+2 .
\end{aligned}
$$

Then $c_{1}(V) \leq-3$ (from Lemma 13) implies

$$
\chi(V(1)) \leq-1
$$

whereas $h^{q}\left(\mathbb{P}^{2}, V(1)\right)=0$ for $q \geq 1$ (from Lemma 14) implies the contradictory

$$
\chi(V(1))=h^{0}\left(\mathbb{P}^{2}, V(1)\right) \geq 0 .
$$

Corollary 16 The four-fold $S_{1} \times S_{2}$ given by the product of two K3 surfaces cannot be fibred by abelian surfaces over $\mathbb{P}^{2}$.

In the second subcase, recall that $X=S_{1} \times S_{2} /\left\langle\left(\gamma_{1}, \gamma_{2}\right)\right\rangle$ by Proposition 5 , where $\gamma_{1}$ is the covering involution of the double cover $S_{1} \rightarrow T_{1}$ of an Enriques surface and $\gamma_{2}$ is the covering involution of some other (possibly ramified) double cover $S_{2} \rightarrow T_{2}$.

Proposition 17 The four-fold $X=S_{1} \times S_{2} /\left\langle\left(\gamma_{1}, \gamma_{2}\right)\right\rangle$ cannot be fibred by abelian surfaces over $\mathbb{P}^{2}$.

Proof Suppose we have a fibration $X \rightarrow B$ by abelian surfaces, with $B$ some surface, and let $A$ be a generic fibre. By composing the inclusion $A \hookrightarrow X$, the (possibly ramified) double cover

$$
X=S_{1} \times S_{2} /\left\langle\left(\gamma_{1}, \gamma_{2}\right)\right\rangle \rightarrow T_{1} \times T_{2}
$$

and the projection to $T_{1}$ we get a map $A \rightarrow T_{1}$. The Enriques surface has Picard number $\rho\left(T_{1}\right)=10$, whereas the abelian surface has Picard number $\rho(A)$ at most four. Therefore $A \rightarrow T_{1}$ cannot be surjective. 
If the image of $A \rightarrow T_{1}$ were zero-dimensional it would have to be a single point as $A$ is connected. Then the deformations of $A$ in $X$ would be parametrized either by $T_{1}$ or by a double cover of $T_{1}$. In other words, $B$ would be isomorphic either to $T_{1}$ or to a double cover of $T_{1}$; but neither of these is $\mathbb{P}^{2}$ (in the latter case, the Picard number $\left.\rho(B) \geq \rho\left(T_{1}\right)=10\right)$.

Finally, suppose the image of $A \rightarrow T_{1}$ is a curve $C \subset T_{1}$. The base of a fibration $A \rightarrow C$ of an abelian surface over a curve must have genus zero or one. If $C$ were a rational curve it would be rigid in $T_{1}$; but as we move $A$ in $X$ the curve $C$ should also move in $T_{1}$. Therefore $C$ is an elliptic curve, a fibre of an elliptic fibration $T_{1} \rightarrow \mathbb{P}^{1}$ on the Enriques surface. This induces a map $B \rightarrow \mathbb{P}^{1}$ (the point in $B$ corresponding to the fibre $A$ is mapped to the point in $\mathbb{P}^{1}$ corresponding to the fibre $C$ ), and therefore the Picard number $\rho(B) \geq 2$. Again we see that $B$ cannot be $\mathbb{P}^{2}$.

\section{$6 \tilde{X}=E \times Y, E$ an elliptic curve, $Y$ a Calabi-Yau three-fold}

There is only one possibility for $\left(h^{q}\left(\mathbb{P}^{2}, V\right)\right)_{q=0}^{2}=\left(h^{i}\left(X, \mathcal{O}_{X}\right)\right)_{i=1}^{3}$, namely $(1,0,1)$. However, we can't conclude anything about the degree $d$ of the cover $\tilde{X} \rightarrow X$, so $X$ itself does not necessarily decompose as $E \times Y$. By Lemma 12 the bundle $V$ must be isomorphic to $\mathcal{O}_{\mathbb{P}^{2}} \oplus \mathcal{O}_{\mathbb{P}^{2}}(-3)$.

Example An obvious example is given by choosing the Calabi-Yau three-fold $Y$ to be an elliptic fibration over $\mathbb{P}^{2}$, and letting $X=\tilde{X}=E \times Y$. Then $X$ is an abelian surface fibration over $\mathbb{P}^{2}$ whose fibres are products of $E$ and the elliptic fibres of $\eta: Y \rightarrow \mathbb{P}^{2}$. Let us examine $Y$ more closely.

By assumption $X \rightarrow \mathbb{P}^{2}$ has a section $s$, and therefore $Y \rightarrow \mathbb{P}^{2}$ must admit a section too, which we also denote by $s$. Some singular fibres of $Y \rightarrow \mathbb{P}^{2}$ may consist of several irreducible components; by blowing down all components of the singular fibres that the section does not meet, we obtain the Weierstrass model $\bar{\eta}: \bar{Y} \rightarrow \mathbb{P}^{2}$ of $Y$. We recall the description of the Weierstrass model $\bar{Y}$ as a family of plane cubics over $\mathbb{P}^{2}$ (for example, Friedman [6] describes the theory of elliptic surfaces, but his arguments on pages 180-181 apply to general elliptic fibrations).

A single elliptic curve $E$ is embedded as a cubic in the plane by

$$
E \hookrightarrow \mathbb{P}\left(\mathrm{H}^{0}(E, \mathcal{O}(3 p))^{*}\right)
$$

where $p$ is some basepoint on $E$. In the relative version the basepoint in each curve is given by the section $s$, and the embedding looks like

$$
\bar{Y} \hookrightarrow \mathbb{P}\left(W^{*}\right)
$$

where $W$ is the rank three bundle $\bar{\eta}_{*} \mathcal{O}_{\bar{Y}}(3 s)$. One can show that

$$
W \cong L^{-2} \oplus L^{-3} \oplus \mathcal{O}_{\mathbb{P}^{2}}
$$


where $L$ is the dual of the line bundle $R^{1} \bar{\eta}_{*} \mathcal{O}_{\bar{Y}}$. Since $Y$ (and hence $\bar{Y}$ ) has trivial canonical bundle, we must have

$$
R^{1} \bar{\eta}_{*} \mathcal{O}_{\bar{Y}}=R^{1} \eta_{*} \mathcal{O}_{Y} \cong K_{\mathbb{P}^{2}}=\mathcal{O}_{\mathbb{P}^{2}}(-3)
$$

and therefore $L \cong \mathcal{O}_{\mathbb{P}^{2}}(3)$ and

$$
W^{*} \cong \mathcal{O}_{\mathbb{P}^{2}}(6) \oplus \mathcal{O}_{\mathbb{P}^{2}}(9) \oplus \mathcal{O}_{\mathbb{P}^{2}} .
$$

Let $(x, y, z)$ denote fibre coordinates on $W^{*}$ corresponding to the above decomposition. Then $\bar{Y}$ is a family of cubics in $\mathbb{P}\left(W^{*}\right)$ over the projective plane given by the equation

$$
y^{2} z=x^{3}+a x z^{2}+b z^{3},
$$

where $a$ and $b$ are sections of $\mathcal{O}_{\mathbb{P}^{2}}(12)$ and $\mathcal{O}_{\mathbb{P}^{2}}(18)$ respectively. The discriminant locus $\Delta \subset \mathbb{P}^{2}$ parametrizing singular fibres is the zero locus of

$$
4 a^{3}+27 b^{2} \in \mathrm{H}^{0}\left(\mathbb{P}^{2}, \mathcal{O}(36)\right) .
$$

For generic $a$ and $b, \Delta$ is a smooth curve in the plane and every singular fibre is a nodal rational curve (in particular, this means that the Weierstrass model $\bar{Y}$ is smooth and isomorphic to $Y$ itself). It looks like the family of such fibrations $\bar{Y} \rightarrow \mathbb{P}^{2}$ will have dimension

$$
h^{0}\left(\mathbb{P}^{2}, \mathcal{O}(12)\right)+h^{0}\left(\mathbb{P}^{2}, \mathcal{O}(18)\right)=13+19=32,
$$

though actually $a$ and $b$ are only defined up to a rescaling

$$
(a, b) \mapsto\left(\lambda^{4} a, \lambda^{6} b\right)
$$

where $\lambda \in \mathbb{C}^{*}$, and we also need to quotient by the automorphism group $\operatorname{PGL}(3, \mathbb{C})$ of $\mathbb{P}^{2}$. Thus we are left with a family of dimension

$$
32-1-8=23 \text {. }
$$

In addition, the moduli space of elliptic curves is one-dimensional, so $X=E \times Y$ depends on 24 parameters.

Remark Suppose that $X=E \times Y$. The image of $Y_{t}:=\{t\} \times Y$ under the projection $\pi: X \rightarrow \mathbb{P}^{2}$ must be at least one-dimensional. If the image is a curve $C \subset \mathbb{P}^{2}$, then the (trivial) normal bundle of $Y_{t}$ in $E \times Y$ would be the pull-back of the non-trivial normal bundle of $C$ in $\mathbb{P}^{2}$, a contradiction. Therefore $Y_{t} \rightarrow \mathbb{P}^{2}$ is surjective. The fibres of $Y_{t} \rightarrow \mathbb{P}^{2}$ must be connected, since the fibres of $X \rightarrow \mathbb{P}^{2}$ are connected; this makes $Y_{t}$ into an elliptic fibration over $\mathbb{P}^{2}$. Moreover, the morphism to $\mathbb{P}^{2}$ is canonically given by

$$
Y_{t} \rightarrow \mathbb{P}\left(\mathrm{H}^{0}\left(Y_{t}, L_{t}\right)^{*}\right)
$$

where $L_{t}$ is the pull-back of the hyperplane line bundle from $\mathbb{P}^{2}$. Since $Y_{t} \cong Y$ for all $t \in E$, and the line bundle $L_{t}$ cannot be deformed because $\mathrm{H}^{1}(Y, \mathcal{O})=0$, the elliptic fibration $Y_{t} \rightarrow \mathbb{P}^{2}$ will not depend on $t$ either. Therefore $X=E \times Y \rightarrow \mathbb{P}^{2}$ is exactly as constructed in the example, the product of an elliptic curve and an elliptic fibration over $\mathbb{P}^{2}$. cated.

The general situation, when $X$ is a quotient of $\tilde{X}=E \times Y$, is more compli- 


\section{$7 \quad \tilde{X}=X$ a Calabi-Yau four-fold}

There is one possibility for $\left(h^{q}\left(\mathbb{P}^{2}, V\right)\right)_{q=0}^{2}=\left(h^{i}\left(X, \mathcal{O}_{X}\right)\right)_{i=1}^{3}$, namely $(0,0,0)$. By Lemma 13 we know that $c_{1}(V) \leq-3$, and by Lemma 14 we know that $h^{q}\left(\mathbb{P}^{2}, V(k)\right)=0$ for $k \geq 1$ and $q \geq 1$.

Lemma 18 The bundle $V$ on $\mathbb{P}^{2}$ is isomorphic to either $\mathcal{O}_{\mathbb{P}^{2}}(-1) \oplus \mathcal{O}_{\mathbb{P}^{2}}(-2)$ or $\mathcal{O}_{\mathbb{P}^{2}}(-2) \oplus \mathcal{O}_{\mathbb{P}^{2}}(-2)$. Since the latter bundle has first Chern class -4, it cannot occur if either of the hypotheses of Lemma 13 regarding the singular fibres of $X \rightarrow \mathbb{P}^{2}$ are satisfied (i.e., first cohomology at least two-dimensional or unipotent monodromy).

Proof As in the proof of Lemma 15, we can use the Riemann-Roch formula and $\chi(V)=0$ to calculate

$$
\begin{aligned}
\chi(V(1)) & =\chi(V)+c_{1}(V)+4 \\
& =c_{1}(V)+4 .
\end{aligned}
$$

Lemma 14 gives

$$
\chi(V(1))=h^{0}\left(\mathbb{P}^{2}, V(1)\right) \geq 0
$$

and hence $c_{1}(V) \geq-4$. This complements the inequality $c_{1}(V) \leq-3$ of Lemma 13.

First suppose that $c_{1}(V)=-3$; then $h^{0}\left(\mathbb{P}^{2}, V(1)\right)=1$ and we have a morphism $\mathcal{O}_{\mathbb{P}^{2}}(-1) \rightarrow V$. The cokernel of this map is of the form $\mathcal{O}_{\mathbb{P}^{2}}(k) \otimes \mathcal{I}_{Z}$, where $\mathcal{I}_{Z}$ is the ideal sheaf of some zero-dimensional subscheme $Z \subset \mathbb{P}^{2}$. Moreover $k=-2$ because $c_{1}(V)=-3$. Substituting $\chi(V)=0$ and $c_{1}(V)=-3$ into the Riemann-Roch formula gives $c_{2}(V)=2$, which implies that the zero-dimensional subscheme $Z$ is empty. The short exact sequence

$$
0 \rightarrow \mathcal{O}_{\mathbb{P}^{2}}(-1) \rightarrow V \rightarrow \mathcal{O}_{\mathbb{P}^{2}}(-2) \rightarrow 0
$$

must split, giving $V \cong \mathcal{O}_{\mathbb{P}^{2}}(-1) \oplus \mathcal{O}_{\mathbb{P}^{2}}(-2)$.

A similar argument works when $c_{1}(V)=-4$. Although $h^{0}\left(\mathbb{P}^{2}, V(1)\right)$ is now zero, we can instead compute $h^{0}\left(\mathbb{P}^{2}, V(2)\right)$ and find that it equals two. Let the cokernel of the evaluation map

$$
\mathrm{H}^{0}\left(\mathbb{P}^{2}, V(2)\right) \otimes \mathcal{O}_{\mathbb{P}^{2}}(-2) \rightarrow V
$$

be the torsion sheaf $\mathcal{F}$. The Riemann-Roch formula yields $c_{2}(V)=4$, and since the Chern classes of $V$ agree with those of $\mathcal{O}_{\mathbb{P}^{2}}(-2) \oplus \mathcal{O}_{\mathbb{P}^{2}}(-2)$ the evaluation map must be an isomorphism and $\mathcal{F}$ must vanish. Therefore $V \cong \mathcal{O}_{\mathbb{P}^{2}}(-2) \oplus \mathcal{O}_{\mathbb{P}^{2}}(-2)$.

We will describe here an example whose fibres are products of elliptic curves; in Section 9 we will describe an example whose fibres are Jacobians of genus two curves. Both examples will have $V \cong \mathcal{O}_{\mathbb{P}^{2}}(-1) \oplus \mathcal{O}_{\mathbb{P}^{2}}(-2)$. 
Example For $i=1$ and 2, let $\pi_{i}: X_{i} \rightarrow \mathbb{P}^{2}$ be elliptic fibrations with $R^{1} \pi_{i *} \mathcal{O}_{X_{i}} \cong \mathcal{O}_{\mathbb{P}^{2}}(-i)$. For example, we could let $X_{1}$ be a Weierstrass model given by a family of cubics

$$
y^{2} z=x^{3}+a x z^{2}+b z^{3}
$$

in the $\mathbb{P}^{2}$-bundle

$$
\mathbb{P}\left(\mathcal{O}_{\mathbb{P}^{2}}(2) \oplus \mathcal{O}_{\mathbb{P}^{2}}(3) \oplus \mathcal{O}_{\mathbb{P}^{2}}\right)
$$

over the projective plane, where $a$ and $b$ are generic sections of $\mathcal{O}_{\mathbb{P}^{2}}(4)$ and $\mathcal{O}_{\mathbb{P}^{2}}(6)$ respectively. By genericity, the discriminant locus

$$
\Delta_{1}=\left\{4 a^{3}+27 b^{2}=0\right\} \subset \mathbb{P}^{2}
$$

will be a smooth curve; thus the singular fibres will all be nodal rational curves and $X_{1}$ will be smooth. Likewise, we let $X_{2}$ be a Weierstrass model given by

$$
y^{2} z=x^{3}+c x z^{2}+d z^{3}
$$

in

$$
\mathbb{P}\left(\mathcal{O}_{\mathbb{P}^{2}}(4) \oplus \mathcal{O}_{\mathbb{P}^{2}}(6) \oplus \mathcal{O}_{\mathbb{P}^{2}}\right)
$$

where $c$ and $d$ are generic sections of $\mathcal{O}_{\mathbb{P}^{2}}(8)$ and $\mathcal{O}_{\mathbb{P}^{2}}(12)$ respectively. Again, the discriminant locus

$$
\Delta_{2}=\left\{4 c^{3}+27 d^{2}=0\right\} \subset \mathbb{P}^{2}
$$

will be a smooth curve, the singular fibres will all be nodal rational curves, and $X_{2}$ will be smooth.

Next we define $X$ to be the fibre product $X_{1} \times_{\mathbb{P}^{2}} X_{2}$. Then $X$ is a fibration over $\mathbb{P}^{2}$ whose fibres are products of elliptic curves. The singular fibres of $X \rightarrow \mathbb{P}^{2}$ occur over $\Delta_{1} \cup \Delta_{2}$; over $\Delta_{1} \backslash \Delta_{2}$ and $\Delta_{2} \backslash \Delta_{1}$ these are products of nodal rational curves and smooth elliptic curves, whereas over $\Delta_{1} \cap \Delta_{2}$ these are products of two nodal rational curves. By genericity, we can assume that $\Delta_{1}$ and $\Delta_{2}$ intersect transversally (in a collection of points $\Delta_{1} \cap \Delta_{2}$ ), and then a local calculation shows that $X$ is smooth.

Now $\pi: X \rightarrow \mathbb{P}^{2}$ has

$$
V=R^{1} \pi_{*} \mathcal{O}_{X} \cong R^{1} \pi_{1 *} \mathcal{O}_{X_{1}} \oplus R^{1} \pi_{2 *} \mathcal{O}_{X_{2}} \cong \mathcal{O}_{\mathbb{P}^{2}}(-1) \oplus \mathcal{O}_{\mathbb{P}^{2}}(-2) .
$$

The Leray spectral sequence therefore implies that

$$
h^{k}\left(\mathcal{O}_{X}\right)= \begin{cases}1 & \text { if } k=0 \text { or } 4, \\ 0 & \text { otherwise }\end{cases}
$$

We claim that the canonical bundle $K_{X}$ is trivial. Each fibre of $X \rightarrow \mathbb{P}^{2}$ has trivial canonical bundle and trivial normal bundle. Therefore the adjunction formula implies that $K_{X}$ is trivial when restricted to any fibre; but then $K_{X}$ must be the pull-back $\pi^{*} \mathcal{O}_{\mathbb{P}^{2}}(m)$ of a line bundle on $\mathbb{P}^{2}$. Since

$$
h^{0}\left(K_{X}\right)=h^{0}\left(\Omega_{X}^{4}\right)=h^{4}\left(\mathcal{O}_{X}\right)=1,
$$


the canonical bundle $K_{X}$ has a unique section up to scale. This implies that $m=0$, proving the claim.

Finally, $X$ must be a Calabi-Yau four-fold as the only other four-fold $X$ with trivial canonical bundle and $h^{k}\left(\mathcal{O}_{X}\right)$ as above is the quotient of a product $S_{1} \times S_{2}$ of K3 surfaces by an involution, but Proposition 17 says that such a four-fold cannot be fibred by abelian surfaces over $\mathbb{P}^{2}$.

Note that $a, b, c$, and $d$ are defined up to rescalings

$$
(a, b) \mapsto\left(\lambda^{4} a, \lambda^{6} b\right) \quad \text { and } \quad(c, d) \mapsto\left(\mu^{4} c, \mu^{6} d\right)
$$

where $\lambda$ and $\mu$ are in $\mathbb{C}^{*}$. We also need to quotient by the automorphism group of $\mathbb{P}^{2}$, and thus we get a family of examples depending on

$$
\begin{array}{r}
h^{0}\left(\mathbb{P}^{2}, \mathcal{O}(4)\right)+h^{0}\left(\mathbb{P}^{2}, \mathcal{O}(6)\right)+h^{0}\left(\mathbb{P}^{2}, \mathcal{O}(8)\right)+h^{0}\left(\mathbb{P}^{2}, \mathcal{O}(12)\right)-2-\operatorname{dimPGL}(3, \mathbb{C}) \\
=5+7+9+13-2-8=24
\end{array}
$$

parameters.

\section{$8 \tilde{X}=X$ an irreducible holomorphic symplectic four-fold}

There is one possibility for $\left(h^{q}\left(\mathbb{P}^{2}, V\right)\right)_{q=0}^{2}=\left(h^{i}\left(X, \mathcal{O}_{X}\right)\right)_{i=1}^{3}$, namely $(0,1,0)$. Using arguments similar to those above, one can show that $V$ is isomorphic to the cotangent bundle $\Omega^{1}$ on $\mathbb{P}^{2}$ (one obtains a variation of the Euler sequence defining $\Omega^{1}$ ). In fact, $\pi: X \rightarrow \mathbb{P}^{2}$ is a holomorphic Lagrangian fibration, and Matsushita [20] showed more generally that if $\pi: X \rightarrow \mathbb{P}^{n}$ is a Lagrangian fibration on an irreducible holomorphic symplectic $2 n$-fold $X$, then

$$
R^{i} \pi_{*} \mathcal{O}_{X} \cong \Omega_{\mathbb{P} n}^{i}
$$

for all $i=0, \ldots, n$. The isomorphism $V \cong \Omega_{\mathbb{P}^{2}}^{1}$ is a special case of this result.

Up to deformation, there are two known examples of irreducible holomorphic symplectic four-folds. One is the Hilbert scheme $\mathrm{Hilb}^{2} S$ of two points on a K3 surface $S$, which was first described by Fujiki [7] as

$$
\operatorname{Hilb}^{2} S=\left(\text { Blow }_{\Delta} S \times S\right) / \mathbb{Z}_{2} .
$$

The other is the generalized Kummer four-fold, due to Beauville [1].

Example If $S$ is an elliptic K3 surface, then the elliptic fibration $S \rightarrow \mathbb{P}^{1}$ induces a Lagrangian fibration

$$
\operatorname{Hilb}^{2} S \rightarrow \operatorname{Sym}^{2} \mathbb{P}^{1} \cong \mathbb{P}^{2}
$$

whose fibres are products of elliptic curves. Another example is given by choosing $S$ to contain a genus two curve $C$, which will move in a two-dimensional 
linear system $|C| \cong \mathbb{P}^{2}$. Denote this family of curves by $\mathcal{C} \rightarrow \mathbb{P}^{2}$. Then the compactified relative Jacobian

$$
X:=\overline{\operatorname{Jac}}^{0}\left(\mathcal{C} / \mathbb{P}^{2}\right) \rightarrow \mathbb{P}^{2}
$$

is both birational to and a deformation of $\mathrm{Hilb}^{2} S$. This Lagrangian fibration by Jacobians of genus two curves is known as the Beauville-Mukai integrable system [2].

Suppose that $S$ is a generic polarized K3 surface, i.e., Pic $S$ is generated by the polarization $H$. Proposition 7.1 of Hassett and Tschinkel [10] states that the Hilbert scheme Hilb ${ }^{2} S$ is a Lagrangian fibration by abelian surfaces over $\mathbb{P}^{2}$ if and only if the polarization has degree $H^{2}=2 m^{2}$ for some integer $m>1$ (in the case $m=1$ and $H^{2}=2$, Hilb ${ }^{2} S$ is obtained from the Beauville-Mukai integrable system via a Mukai flop). Independently, Markushevich [16 and the author 24 gave an alternate proof of this result, and generalized it to higher dimensions. In general, if $S$ is not generic, $H^{2}=2 \mathrm{~m}^{2}$ will only imply that $\mathrm{Hilb}^{2} S$ is birational to a Lagrangian fibration.

Example Debarre [4] described Lagrangian fibrations on generalized Kummer varieties (see also Example 3.8 in the author's article [22]). In particular, certain generalized Kummer four-folds admit Lagrangian fibrations over $\mathbb{P}^{2}$ whose fibres are abelian surfaces of polarization type $(1,3)$. Note that by Proposition 5.3 of 22] a generalized Kummer variety cannot be fibred by principally polarized abelian varieties, such as Jacobians of curves. Gulbrandsen 9] and Yoshioka 28, investigated exactly which generalized Kummer varieties are Lagrangian fibrations.

\section{Fibrations by Jacobians}

In this final section we construct examples of four-folds with trivial canonical bundles which are fibred over $\mathbb{P}^{2}$ by Jacobians of genus two curves. Moreover, we classify all such four-folds. The results in this section owe a lot to Markushevich's papers 14, 15]; indeed, the examples we describe are the Beauville-Mukai system (a holomorphic symplectic four-fold) and Example 1 from [14 (which we prove is a Calabi-Yau four-fold). Markushevich [15] classified all irreducible holomorphic symplectic four-folds fibred by Jacobians; our classification result is an extension of his, and uses similar arguments.

We start by defining exactly what we mean by a fibration by Jacobians. The following is essentially Definition 4 from [14].

Definition Let $\mathcal{C} \rightarrow \mathbb{P}^{2}$ be a flat family of irreducible and reduced genus two curves over the projective plane. We say that $\mathcal{C} / \mathbb{P}^{2}$ has mild degenerations if

1. the total space of $\mathcal{C}$ is smooth,

2. the singular curves of the family have only nodes or cusps as singularities, 
3. if $C_{t}$ is a singular curve with two distinct singular points $p_{1}$ and $p_{2}$, then the analytic germs $\left(\Delta_{1}, t\right)$ and $\left(\Delta_{2}, t\right)$ of the discriminant curves of the unfoldings of $\left(C_{t}, p_{1}\right)$ and $\left(C_{t}, p_{2}\right)$ induced by the family $\mathcal{C} / \mathbb{P}^{2}$ must have different reduced tangent cones at $t$.

Remark The smoothness of the total space of $\mathcal{C}$ implies that a generic singular curve will have a single node, and will occur in codimension one in the family. In codimension two we may get curves with cusps or a pair of nodes. The third condition means the following. If $C_{t}$ has nodes at $p_{1}$ and $p_{2}$ then there will be two branches of the discriminant curve $\Delta \subset \mathbb{P}^{2}$ at $t$. Along one branch, the curves will have nodes whose limit is $p_{1}$ as we approach $C_{t}$, and along the other branch the curves will have nodes whose limit is $p_{2}$. The mild degenerations condition requires these two branches to meet transversally at $t$.

Definition By a fibration by Jacobians over $\mathbb{P}^{2}$ we will mean the compactified relative Jacobian

$$
X:=\overline{\operatorname{Jac}}^{0}\left(\mathcal{C} / \mathbb{P}^{2}\right)
$$

of a family $\mathcal{C} / \mathbb{P}^{2}$ of irreducible and reduced genus two curves with mild degenerations.

Remark Theorem 2 of [14] states that if $\mathcal{C} / \mathbb{P}^{2}$ has mild degenerations then its compactified relative Jacobian $X$ is smooth and its canonical bundle $K_{X}$ is trivial when restricted to the fibres of $X \rightarrow \mathbb{P}^{2}$. Markushevich strengthened this theorem in 15 , by giving necessary and sufficient conditions on a family of curves for its compactified relative Jacobian to be smooth. We will stick with the mild degenerations condition for simplicity.

Lemma 19 Let $\pi: X \rightarrow \mathbb{P}^{2}$ be a fibrations by Jacobians over $\mathbb{P}^{2}$ constructed from a family of curves $\mathcal{C} / \mathbb{P}^{2}$. Let $Y$ denote the total space of $\mathcal{C} / \mathbb{P}^{2}$. Then $Y$ is a branched double cover of the $\mathbb{P}^{1}$-bundle $\mathbb{P}\left(R^{1} \pi_{*} \mathcal{O}_{X}\right)$ over $\mathbb{P}^{2}$.

Proof A genus two curve $C$ is hyperelliptic: the canonical map

$$
C \rightarrow \mathbb{P}\left(\mathrm{H}^{0}\left(C, K_{C}\right)^{*}\right) \cong \mathbb{P}\left(\mathrm{H}^{1}\left(C, \mathcal{O}_{C}\right)\right)
$$

expresses $C$ as a double cover of $\mathbb{P}^{1}$, branched over six points. The relative version of this expresses $Y$ as a double cover of the $\mathbb{P}^{1}$-bundle $\mathbb{P}\left(R^{1} f_{*} \mathcal{O}_{Y}\right)$ over $\mathbb{P}^{2}$, branched over a divisor which intersects each fibre $\mathbb{P}^{1}$ in six points, counted with multiplicity. Here $f$ denotes the projection $Y \rightarrow \mathbb{P}^{2}$.

It remains to show that

$$
R^{1} f_{*} \mathcal{O}_{Y} \cong R^{1} \pi_{*} \mathcal{O}_{X}
$$

but this is Lemma 5 in [26]. More precisely, Lemma 5 is for Lagrangian fibrations, but the same argument works provided that the generic singular curves of $\mathcal{C} / \mathbb{P}^{2}$ have only single nodes, which is guaranteed by the mild degenerations hypothesis. 
We now reverse the construction by starting with a rank two bundle $W$ on $\mathbb{P}^{2}$. Let $Y$ be a double cover of the $\mathbb{P}^{1}$-bundle $h: \mathbb{P}(W) \rightarrow \mathbb{P}^{2}$ branched over a divisor corresponding to a generic section of

$$
\mathcal{O}_{\mathbb{P}(W)}(6) \otimes h^{*} \mathcal{O}(2 d) .
$$

Such a divisor will intersect each fibre $\mathbb{P}^{1}$ in six points, counted with multiplicity, and hence $f: Y \rightarrow \mathbb{P}^{2}$ is a family of genus two curves. Assume this family has mild degenerations and let $\pi: X \rightarrow \mathbb{P}^{2}$ be the compactified relative Jacobian of $Y / \mathbb{P}^{2}$. The previous lemma says that $W$ must be isomorphic to

$$
R^{1} f_{*} \mathcal{O}_{Y} \cong R^{1} \pi_{*} \mathcal{O}_{X}
$$

up to tensoring with a line bundle. We can identify this line bundle in terms of the branch locus of the double cover. This is essentially Proposition 5 of Markushevich [14, though we avoid local coordinates in our proof.

Lemma 20 In the above situation

$$
W \cong \mathcal{O}(d) \otimes \Lambda^{2} W^{*} \otimes R^{1} f_{*} \mathcal{O}_{Y} .
$$

Proof Denote the double cover by $g: Y \rightarrow \mathbb{P}(W)$. We have a commutative diagram

$$
\begin{aligned}
Y \quad \stackrel{g}{\rightarrow} & \mathbb{P}(W) \\
f \searrow & \downarrow h \\
& \mathbb{P}^{2} .
\end{aligned}
$$

Then

$$
R^{1} f_{*} \mathcal{O}_{Y} \cong R^{1}(h \circ g)_{*} \mathcal{O}_{Y} \cong R^{1} h_{*} \circ R^{0} g_{*} \mathcal{O}_{Y}
$$

since $g$ is a finite map and therefore $R^{1} g_{*} \mathcal{O}_{Y}$ vanishes. Because of the choice of branch divisor

$$
R^{0} g_{*} \mathcal{O}_{Y} \cong \mathcal{O}_{\mathbb{P}(W)} \oplus\left(\mathcal{O}_{\mathbb{P}(W)}(-3) \otimes h^{*} \mathcal{O}(-d)\right),
$$

and thus

$$
\begin{aligned}
R^{1} f_{*} \mathcal{O}_{Y} & \cong R^{1} h_{*} \mathcal{O}_{\mathbb{P}(W)} \oplus R^{1} h_{*}\left(\mathcal{O}_{\mathbb{P}(W)}(-3) \otimes h^{*} \mathcal{O}(-d)\right) \\
& \cong \mathcal{O}(-d) \otimes R^{1} h_{*} \mathcal{O}_{\mathbb{P}(W)}(-3) \\
& \cong \mathcal{O}(-d) \otimes\left(R^{0} h_{*} \omega_{h}(3)\right)^{*}
\end{aligned}
$$

where on the second line we have used the fact that $R^{1} h_{*} \mathcal{O}_{\mathbb{P}(W)}$ vanishes (the fibres of $h: \mathbb{P}(W) \rightarrow \mathbb{P}^{2}$ are rational curves) and on the third line we have used relative Serre duality. The relative dualizing sheaf $\omega_{h}$ of $h$ is given by

$$
\omega_{h} \cong \mathcal{O}_{\mathbb{P}(W)}(-2) \otimes h^{*} \Lambda^{2} W^{*} .
$$

Inserting this in the above equation gives

$$
\begin{aligned}
R^{1} f_{*} \mathcal{O}_{Y} & \cong \mathcal{O}(-d) \otimes\left(R^{0} h_{*}\left(\mathcal{O}_{\mathbb{P}(W)}(1) \otimes h^{*} \Lambda^{2} W^{*}\right)\right)^{*} \\
& \cong \mathcal{O}(-d) \otimes \Lambda^{2} W \otimes\left(R^{0} h_{*} \mathcal{O}_{\mathbb{P}(W)}(1)\right)^{*} \\
& \cong \mathcal{O}(-d) \otimes \Lambda^{2} W \otimes W
\end{aligned}
$$

and the statement of the lemma follows. 
We have already established which rank two vector bundles can arise as $R^{1} \pi_{*} \mathcal{O}_{X}$ for a four-fold with trivial canonical bundle $X$ fibred over $\mathbb{P}^{2}$ by abelian surfaces. Recall that $R^{1} \pi_{*} \mathcal{O}_{X}$ must be one of the following:

1. $\mathcal{O}_{\mathbb{P}^{2}} \oplus \mathcal{O}_{\mathbb{P}^{2}}(-3)$ if the finite cover $\tilde{X}$ of $X$ is either an abelian four-fold, a product $A \times S$ of abelian and K3 surfaces, or a product $E \times Y$ of an elliptic curve and a Calabi-Yau three-fold,

2. $\mathcal{O}_{\mathbb{P}^{2}}(-1) \oplus \mathcal{O}_{\mathbb{P}^{2}}(-2)$ or $\mathcal{O}_{\mathbb{P}^{2}}(-2) \oplus \mathcal{O}_{\mathbb{P}^{2}}(-2)$ if $\tilde{X}$ is a Calabi-Yau four-fold,

3. $\Omega_{\mathbb{P}^{2}}^{1}$ if $\tilde{X}=X$ is an irreducible holomorphic symplectic four-fold.

If $X$ is the compactified relative Jacobian of a family of curves with mild degenerations, then the generic singular fibre of $X \rightarrow \mathbb{P}^{2}$ will be the compactified Jacobian of a curve with a single node. In this case, both of the conditions in Lemma 13 will be satisfied, implying $c_{1}\left(R^{1} \pi_{*} \mathcal{O}_{X}\right)=-3$. Thus $\mathcal{O}_{\mathbb{P}^{2}}(-2) \oplus \mathcal{O}_{\mathbb{P}^{2}}(-2)$ is impossible; we are left with the three other possibilities.

Returning to the construction of the family of curves, $W$ must be isomorphic to $R^{1} f_{*} \mathcal{O}_{Y}$ up to tensoring with a line bundle. We can normalize the first Chern class of $W$ by assuming this line bundle is trivial, i.e., we set

$$
W \cong R^{1} f_{*} \mathcal{O}_{Y} \cong R^{1} \pi_{*} \mathcal{O}_{X}
$$

The isomorphism

$$
W \cong \mathcal{O}(d) \otimes \Lambda^{2} W^{*} \otimes R^{1} f_{*} \mathcal{O}_{Y}
$$

of Lemma 20 then yields

$$
d=c_{1}(W)=c_{1}\left(R^{1} f_{*} \mathcal{O}_{Y}\right)=c_{1}\left(R^{1} \pi_{*} \mathcal{O}_{X}\right)=-3,
$$

and hence the branch divisor is the zero locus of a generic section of

$$
\mathcal{O}_{\mathbb{P}(W)}(6) \otimes h^{*} \mathcal{O}(-6)
$$

on $\mathbb{P}(W)$. Now

$$
\begin{aligned}
\mathrm{H}^{0}\left(\mathbb{P}(W), \mathcal{O}_{\mathbb{P}(W)}(6) \otimes h^{*} \mathcal{O}(-6)\right) & \cong \mathrm{H}^{0}\left(\mathbb{P}^{2}, R^{0} h_{*}\left(\mathcal{O}_{\mathbb{P}(W)}(6) \otimes h^{*} \mathcal{O}(-6)\right)\right) \\
& \cong \mathrm{H}^{0}\left(\mathbb{P}^{2}, \mathcal{O}(-6) \otimes R^{0} h_{*} \mathcal{O}_{\mathbb{P}(W)}(6)\right) \\
& \cong \mathrm{H}^{0}\left(\mathbb{P}^{2}, \mathcal{O}(-6) \otimes \operatorname{Sym}^{6} W^{*}\right)
\end{aligned}
$$

We consider the three possibilities for $W \cong R^{1} f_{*} \mathcal{O}_{Y} \cong R^{1} \pi_{*} \mathcal{O}_{X}$.

Proposition 21 The case $W \cong \mathcal{O}_{\mathbb{P}^{2}} \oplus \mathcal{O}_{\mathbb{P}^{2}}(-3)$ is impossible. In particular, if the finite cover $\tilde{X}$ of $X$ is either an abelian four-fold, a product $A \times S$ of abelian and $K 3$ surfaces, or a product $E \times Y$ of an elliptic curve and a Calabi-Yau three-fold then $X$ cannot be a fibration by Jacobians over $\mathbb{P}^{2}$. 
Proof If $W \cong \mathcal{O}_{\mathbb{P}^{2}} \oplus \mathcal{O}_{\mathbb{P}^{2}}(-3)$ then the branch divisor of $Y \rightarrow \mathbb{P}(W)$ corresponds to a section in

$$
\mathrm{H}^{0}\left(\mathbb{P}^{2}, \mathcal{O}(-6) \otimes \operatorname{Sym}^{6} W^{*}\right) \cong \mathrm{H}^{0}\left(\mathbb{P}^{2}, \mathcal{O}(-6) \oplus \mathcal{O}(-3) \oplus \ldots \oplus \mathcal{O}(12)\right) .
$$

If we write this section as $\left(s_{0}, s_{1}, \ldots, s_{6}\right)$ then $s_{0}$ and $s_{1}$ must vanish, since they are sections of line bundles of negative degree. Let us consider what this means.

A fibre $Y_{t}$ of $Y \rightarrow \mathbb{P}^{2}$ is a genus two curve, which is a double cover of $\mathbb{P}\left(W_{t}\right)$ branched over six points. Each point corresponds to a line in $W_{t}^{*}$, and therefore the six points define a line in $\operatorname{Sym}^{6} W_{t}^{*}$. This is the same as the line in $\operatorname{Sym}^{6} W_{t}^{*}$ given by evaluating the section $\left(s_{0}, s_{1}, \ldots, s_{6}\right)$ at $t$, since the branch locus is defined by this section. We recover the six points from $\left(s_{0}, s_{1}, \ldots, s_{6}\right)$ by taking the roots of the polynomial

$$
s_{6} z^{6}+s_{5} z^{5}+\ldots+s_{1} z+s_{0} .
$$

If $s_{0}$ and $s_{1}$ vanish at $t$, then $z=0$ is a repeated root. This means that two of the branch points coincide and the genus two curve $Y_{t}$ is singular. If $s_{0}$ and $s_{1}$ vanish identically, then all curves $Y_{t}$ in the family $Y \rightarrow \mathbb{P}^{2}$ are singular. This contradicts the mild degenerations assumption.

Proposition 22 The case $W \cong \mathcal{O}_{\mathbb{P}^{2}}(-1) \oplus \mathcal{O}_{\mathbb{P}^{2}}(-2)$ produces a Calabi-Yau four-fold $X$. This example belongs to a 75-dimensional family.

Remark Markushevich described this four-fold in Example 1 of [14. Our results enable us to identify it as a Calabi-Yau four-fold.

Proof If $W \cong \mathcal{O}_{\mathbb{P}^{2}}(-1) \oplus \mathcal{O}_{\mathbb{P}^{2}}(-2)$ then the branch divisor of $Y \rightarrow \mathbb{P}(W)$ corresponds to a section in

$$
\mathrm{H}^{0}\left(\mathbb{P}^{2}, \mathcal{O}(-6) \otimes \operatorname{Sym}^{6} W^{*}\right) \cong \mathrm{H}^{0}\left(\mathbb{P}^{2}, \mathcal{O} \oplus \mathcal{O}(1) \oplus \ldots \oplus \mathcal{O}(6)\right) .
$$

If this section is chosen generically then the resulting family of genus two curves $Y \rightarrow \mathbb{P}^{2}$ will have mild degenerations, and hence by Theorem 2 of Markushevich 14 its compactified relative Jacobian $X$ will be a smooth four-fold whose canonical bundle $K_{X}$ is trivial when restricted to the fibres of $\pi: X \rightarrow \mathbb{P}^{2}$. In particular, $K_{X}$ is the pull-back $\pi^{*} \mathcal{O}_{\mathbb{P}^{2}}(m)$ of a line bundle on $\mathbb{P}^{2}$. We can proceed as in the example of Section 7: substituting

$$
R^{1} \pi_{*} \mathcal{O}_{X} \cong W \cong \mathcal{O}_{\mathbb{P}^{2}}(-1) \oplus \mathcal{O}_{\mathbb{P}^{2}}(-2)
$$

into the Leray spectral sequence gives

$$
h^{k}\left(\mathcal{O}_{X}\right)= \begin{cases}1 & \text { if } k=0 \text { or } 4 \\ 0 & \text { otherwise }\end{cases}
$$

Therefore $m=0$ and $K_{X}$ is trivial. A four-fold $X$ with trivial canonical bundle and these Hodge numbers must be a Calabi-Yau four-fold or the quotient of 
a product $S_{1} \times S_{2}$ of K3 surfaces by an involution, but by Proposition 17 the latter cannot be fibred by abelian surfaces over $\mathbb{P}^{2}$.

The space of sections above has dimension

$$
1+3+6+10+15+21+28=84 .
$$

Projectivizing and quotienting by the automorphism group $\mathrm{PGL}(3, \mathbb{C})$ of $\mathbb{P}^{2}$ leaves

$$
84-1-8=75
$$

parameters.

The final result of this section is really Theorem 5 of Markushevich [14; we include a slight simplification of his proof, which avoids calculations in local coordinates.

Proposition 23 The case $W \cong \Omega_{\mathbb{P}^{2}}^{1}$ produces an irreducible holomorphic symplectic four-fold $X$. Moreover, $X$ is isomorphic to the Beauville-Mukai system, i.e., the family of genus two curves is a complete linear system of curves on a K3 surface $S$. In particular, $X$ is deformation equivalent to $\mathrm{Hilb}^{2} S$. This example belongs to a 19-dimensional family.

Proof If $W \cong \Omega_{\mathbb{P}^{2}}^{1}$ then the branch divisor of $Y \rightarrow \mathbb{P}(W)$ corresponds to a section in

$$
\mathrm{H}^{0}\left(\mathbb{P}^{2}, \mathcal{O}(-6) \otimes \operatorname{Sym}^{6} W^{*}\right) \cong \mathrm{H}^{0}\left(\mathbb{P}^{2}, \mathcal{O}(-6) \otimes \operatorname{Sym}^{6} T\right) .
$$

As in the previous proposition, a generic choice of section produces a family of curves with mild degenerations whose compactified relative Jacobian $X$ is a smooth four-fold whose canonical bundle $K_{X}$ is the pull-back of a line bundle on $\mathbb{P}^{2}$. Since

$$
R^{1} \pi_{*} \mathcal{O}_{X} \cong W \cong \Omega_{\mathbb{P}^{2}}^{1}
$$

the Leray spectral sequence gives

$$
h^{k}\left(\mathcal{O}_{X}\right)= \begin{cases}1 & \text { if } k=0,2, \text { or } 4, \\ 0 & \text { otherwise }\end{cases}
$$

As before, we can argue that $K_{X}$ is trivial. Then the holonomy classification (Proposition 4) implies that $X$ is an irreducible holomorphic symplectic fourfold. In particular, the generator $\sigma$ of the one-dimensional space

$$
\mathrm{H}^{0}\left(\Omega_{X}^{2}\right) \cong \mathrm{H}^{2}\left(\mathcal{O}_{X}\right)
$$

is a holomorphic symplectic form, and $X$ is simply connected.

Next we calculate the dimension of the space of sections

$$
\mathrm{H}^{0}\left(\mathbb{P}^{2}, \mathcal{O}(-6) \otimes \operatorname{Sym}^{6} T\right) .
$$


Writing $\mathbb{P}^{2}$ as a symmetric space $\mathrm{GL}(3, \mathbb{C}) / P$, the homogeneous bundle

$$
\mathcal{O}(-6) \otimes \operatorname{Sym}^{6} T
$$

corresponds to the $P$-representation $(0 \mid 0,6)$ (see Eastwood and Sawon [5] for notation). The Borel-Weil Theorem then implies that

$$
\mathrm{H}^{0}\left(\mathbb{P}^{2}, \mathcal{O}(-6) \otimes \operatorname{Sym}^{6} T\right)
$$

is isomorphic to the $\mathrm{GL}(3, \mathbb{C})$-representation

$$
(0,0,6) \cong \operatorname{Sym}^{6} \mathbb{C}^{3} .
$$

This has dimension 28. After subtracting one (rescaling of the section) and eight (quotienting by the action of $\operatorname{PGL}(3, \mathbb{C})$ ), we are left with 19 parameters for this family of examples.

Finally, we relate this example to the Beauville-Mukai system. Observe that

$$
\mathbb{P}(W) \cong \mathbb{P}\left(\Omega_{\mathbb{P}^{2}}^{1}\right)
$$

is the incidence subvariety in $\mathbb{P}^{2} \times\left(\mathbb{P}^{2}\right)^{*}$ : a point $q$ in $\left(\mathbb{P}^{2}\right)^{*}$ corresponds to a line in $\mathbb{P}^{2}$, and $(p, q) \in \mathbb{P}(W)$ if and only if $p$ lies on this line. We previously used the isomorphism

$$
\mathrm{H}^{0}\left(\mathbb{P}(W), \mathcal{O}_{\mathbb{P}(W)}(6) \otimes h^{*} \mathcal{O}(-6)\right) \cong \mathrm{H}^{0}\left(\mathbb{P}^{2}, \mathcal{O}(-6) \otimes \operatorname{Sym}^{6} W^{*}\right) .
$$

However, in this example there is also a projection $\mathbb{P}(W) \rightarrow\left(\mathbb{P}^{2}\right)^{*}$. We claim that the line bundle

$$
\mathcal{O}_{\mathbb{P}(W)}(6) \otimes h^{*} \mathcal{O}(-6)
$$

on $\mathbb{P}(W)$ is the pull-back of the line bundle $\mathcal{O}_{\left(\mathbb{P}^{2}\right)^{*}}(6)$ on $\left(\mathbb{P}^{2}\right)^{*}$. This follows directly from the fact that $\mathcal{O}_{\mathbb{P}(W)}(1)$ is the restriction to

$$
\mathbb{P}(W) \subset \mathbb{P}^{2} \times\left(\mathbb{P}^{2}\right)^{*}
$$

of the line bundle $\mathcal{O}_{\mathbb{P}^{2}}(1) \otimes \mathcal{O}_{\left(\mathbb{P}^{2}\right)^{*}}(1)$ on $\mathbb{P}^{2} \times\left(\mathbb{P}^{2}\right)^{*}$. Therefore

$$
\mathrm{H}^{0}\left(\mathbb{P}(W), \mathcal{O}_{\mathbb{P}(W)}(6) \otimes h^{*} \mathcal{O}(-6)\right) \cong \mathrm{H}^{0}\left(\left(\mathbb{P}^{2}\right)^{*}, \mathcal{O}_{\left(\mathbb{P}^{2}\right)^{*}}(6)\right)
$$

and the branch divisor is the pull-back of a sextic curve in $\left(\mathbb{P}^{2}\right)^{*}$.

Let $S$ be the double cover of $\left(\mathbb{P}^{2}\right)^{*}$ branched over the sextic. For a generic choice of sextic, $S$ will be a (smooth) K3 surface; $Y$ will be a $\mathbb{P}^{1}$-bundle over $S$. We have the following commutative diagram.

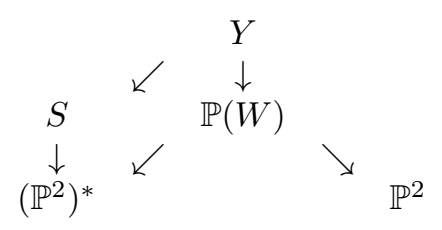


If $t \in \mathbb{P}^{2}$ then $Y_{t}$ is a genus two curve which is a double cover of $\mathbb{P}\left(W_{t}\right)$. The line $\mathbb{P}\left(W_{t}\right)$ projects isomorphically to a line in $\left(\mathbb{P}^{2}\right)^{*}$, whose preimage in $S$ will be a curve isomorphic to $Y_{t}$. Therefore the family of curves $Y \rightarrow \mathbb{P}^{2}$ is (isomorphic to) a complete linear system of curves in the K3 surface $S$. This completes the proof.

Remark In Section 6 of [15], Markushevich described a method of calculating

$$
\mathrm{H}^{0}\left(B, K_{B}^{2} \otimes \operatorname{Sym}^{6} T_{B}\right)
$$

for a toric variety $B$. This was important because he used this method to show that $\mathbb{P}^{2}$ is the only rational surface that can arise as the base of a fibration on an irreducible holomorphic symplectic four-fold. However, later results of Matsushita 17] allow us to conclude more directly that the base must be $\mathbb{P}^{2}$, so we instead use the Borel-Weil Theorem to calculate the above space of sections.

Remark If $S$ is a double cover of the projective plane branched over a sextic, then it has a polarization $H$ with $H^{2}=2$. The moduli space of polarized K3 surfaces with $H^{2}=2$ is 19-dimensional. For each of these we get a BeauvilleMukai system

$$
X:=\overline{\operatorname{Jac}}^{0}\left(\mathcal{C} / \mathbb{P}^{2}\right) .
$$

Note that the dimension of this family of examples agrees with that computed in Proposition 23.

\section{References}

[1] A. Beauville, Variétés Kähleriennes dont la première classe de Chern est nulle, J. Differential Geom. 18 (1983), 755-782.

[2] A. Beauville, Counting rational curves on K3 surfaces, Duke Math. J. 97 (1999), no. 1, 99-108.

[3] F. Bogomolov, On the decomposition of Kähler manifolds with trivial canonical class, Mat. USSR Sbornik 22 (1974), no. 4, 580-583.

[4] O. Debarre, On the Euler characteristic of generalized Kummer varieties, Amer. J. Math. 121 (1999), no. 3, 577-586.

[5] M. Eastwood and J. Sawon, The Borel-Weil theorem for complex projective space, in Invitations to Geometry and Topology, Oxford University Press (2002), 126-145.

[6] R. Friedman, Algebraic surfaces and holomorphic vector bundles, Universitext, Springer-Verlag, New York, 1998.

[7] A. Fujiki, On primitively symplectic compact Kähler $V$-manifolds of dimension four, in Classification of algebraic and analytic manifolds (Katata, 1982), 71-250, Progr. Math. 39 Birkhäuser, 1983. 
[8] O. Fujino and S. Mori, A canonical bundle formula, J. Differential Geom. 56 (2000), no. 1, 167-188.

[9] M. Gulbrandsen, Lagrangian fibrations on generalized Kummer varieties, Bull. Soc. Math. France 135 (2007), no. 2, 283-298.

[10] B. Hassett and Y. Tschinkel, Abelian fibrations and rational points on symmetric products. Internat. J. Math. 11 (2000), no. 9, 1163-1176.

[11] D. Joyce, Compact manifolds with special holonomy, Oxford Mathematical Monographs, Oxford University Press, 2000.

[12] J. Kollár, Higher direct images of dualizing sheaves I, Ann. of Math., 123 (1986), 11-42.

[13] J. Kollár, Higher direct images of dualizing sheaves II, Ann. of Math., 124 (1986), 171-202.

[14] D. Markushevich, Completely integrable projective symplectic 4dimensional varieties, Izvestiya: Mathematics 59 (1995), no. 1, 159187.

[15] D. Markushevich, Lagrangian families of Jacobians of genus 2 curves, J. Math. Sci. 82 (1996), no. 1, 3268-3284.

[16] D. Markushevich, Rational Lagrangian fibrations on punctual Hilbert schemes of K3 surfaces, Manuscripta Math. 120 (2006), no. 2, 131-150.

[17] D. Matsushita, On fibre space structures of a projective irreducible symplectic manifold, Topology 38 (1999), no. 1, 79-83. Addendum, Topology 40 (2001), no. 2, 431-432.

[18] D. Matsushita, Equidimensionality of Lagrangian fibrations on holomorphic symplectic manifolds, Math. Res. Lett. 7 (2000), no. 4, 389-391.

[19] D. Matsushita, Higher direct images of Lagrangian fibrations, preprint math.AG/0010283.

[20] D. Matsushita, Higher direct images of dualizing sheaves of Lagrangian fibrations, Amer. J. Math. 127 (2005), no. 2, 243-259.

[21] K. Oguiso, Shioda-Tate formula for an abelian fibered variety and applications, preprint math.AG/0703245.

[22] J. Sawon, Abelian fibred holomorphic symplectic manifolds, Turkish Jour. Math. 27 (2003), no. 1, 197-230.

[23] J. Sawon, Derived equivalence of holomorphic symplectic manifolds, in Algebraic structures and moduli spaces, 193-211, CRM Proc. Lecture Notes 38, AMS, 2004. 
[24] J. Sawon, Lagrangian fibrations on Hilbert schemes of points on K3 surfaces, J. Algebraic Geom. 16 (2007), no. 3, 477-497.

[25] J. Sawon, Twisted Fourier-Mukai transforms for holomorphic symplectic four-folds, Adv. Math. 218 (2008), no. 3, 828-864.

[26] J. Sawon, A classification of Lagrangian fibrations by Jacobians, preprint arXiv:0803.1186

[27] J. Sawon, Deformations of holomorphic Lagrangian fibrations, Proc. Amer. Math. Soc. 137 (2009), no. 1, 279-285.

[28] K. Yoshioka, Fourier-Mukai transform on abelian surfaces, preprint math.AG/0605190.

Department of Mathematics

sawon@math.colostate.edu

Colorado State University

Fort Collins CO 80523-1874

USA 